\title{
A Hybrid Nature-Inspired Classification Technique for Medical Diagnosis
}

\author{
Suman Muwal \\ Department of Computer \\ Science and Engineering \\ Guru Jambheshwar \\ University of Science and Technology \\ Technology \\ Hisar, Haryana, India
}

\author{
Narender Kumar \\ Department of Computer \\ Science and Engineering \\ Guru Jambheshwar \\ University of Science and \\ Hisar, Haryana, India
}

\begin{abstract}
Early detection of heart disease is essential in medical system because heart disease is the major cause of decease both for men and women. For the medical diagnosis, numerous soft computing techniques are available like Ant Colony Optimization, Genetic Algorithm, Particle Swarm Optimization, Artificial Bee Colony, Firefly Algorithm, Cuckoo Search, Levy Flight etc. The combination of all these evolutionary techniques with the other techniques like artificial neural network, rough set, fuzzy logic and etc. are also possible. The proposed algorithm uses a rough set based attribute reduction with firefly-levy algorithm and the fuzzy logic system for heart disease detection. The combination of these techniques is used to handle the dataset with high dimension and uncertainties. The attribute reduction method is used with the firefly-levy flight algorithm. This will reduce the burden and enhance the performance of classifier. The experiment results show a considerable supremacy of proposed algorithm when compared with other artificial intelligence techniques.
\end{abstract}

\section{Keywords}

Feature Selection, Attribute Reduction, Rough Sets, Firefly Algorithm, Levy Flight Algorithm, Type-2 Fuzzy logic System

\section{INTRODUCTION}

Different features of heart disease may cause the improper functioning of heart. Therefore, fast and accurate detection of heart disease at early stage is necessary. In today's scenario, the soft computing techniques are widely used. Various researches show that the combination of different types of soft computing techniques for hybrid model is mainly used rather than a single technique. Data contains many redundant and irrelevant features that affect the classification process and to solve this problem removal of these features is necessary. Hybrid model have two parts: one for the feature selection and second for the classification.

Nguyen Cong Long et al. [1] proposed a hybrid model which included the attribute reduction based on rough set and the firefly algorithm for feature selection and the interval type-2 fuzzy logic for classification. The interval type-2 fuzzy logic classification is used to handle the large amount of data. For fine-tuning of parameter, chaos firefly algorithm is used by the interval type- 2 fuzzy logic. This model reduced the computational problem and enhanced the performance. The attribute reduction method was compared with the binary particle swarm optimization with rough set based attribute reduction and the IT2FLS classifier was compared with other classifiers like SVM, naïve bayes and ANN. The result demonstrated that the chaos firefly rough set based attribute reduction found the minimal number of attributes from the large data set which helped in the improvement of performance of classification system. The combination of rough set based attribute reduction and interval type-2 fuzzy logic system incapacitates others techniques in terms of accuracy, processing time and convergence speed. The limitation of this approach was that rough set based attribute reduction was not manageable when data contains large number of records and slow training time of type-2 fuzzy logic system. H. Hannah Inbarani et al [2] proposed a hybrid model which integrated the rough set with particle swarm optimization for medical datasets classification. In this study, to find the minimal set of attributes, the relative reduction of attributes based on PSO and the quick reduction algorithm based on PSO was used and obtained results were used as input to classifier. Different types of classifiers were used for classification like Naïve Bayer, Bayes Net and KStar. The results indicated that the attribute reduction by the PSO based relative reduct and the PSO based quick reduct both have 15 out of 44 attributes. The greatest accuracy was $88 \%$ with Naïve Bayer classifier for SPECTF dataset. Disadvantage of this approach was that it did not select minimal number of features.

Arpit Bhardwaj and Aruna Tiwari [3] proposed a combined algorithm of Genetic Algorithm and artificial neural network for classification of the breast cancer. In this paper, modified crossover and mutation operator were used because of the destructive nature of simple crossover and mutation operators. Destructive nature is the problem in GP in which the descendants of good parenthoods generally have low performance than the parents. GONN algorithm was used to classify the breast cancer whether caused death or not death. To authenticate that GONN was superior to other algorithms, different performance measures were used like accuracy, sensitivity, specificity, ROC curve, confusion matrix and AUC under ROC curve. In this paper, there were certain rules in the function set and in the terminal to evolve ANN with GP. Data is divided into training and testing data with four dissimilar panels. The GONN model is compared with the BPNN and Koza and Rice [1991] model. GONN have the uppermost accuracy of $98.24 \%, 99.63 \%, 100 \%$ for $50-50,60$ 40 and $70 \%$ training dataset and $30 \%$ test dataset partitions respectively and 10-fold cross-validation for 50 Genetic programming runs for 1-1-1 neural network as $100 \%$ accuracy. Limitation of this approach was that only crossover and mutation operators were improved and other areas like 
feature selection and feature extraction remained untouched. Also this approach was applied on two class problems only.

Shashikala Mishra et al. [4] presented a novel metaheuristic Bat-inspired classification algorithm for microarray data. Bat algorithm is one of the members of the metaheuristic family which solves the multi objective engineering problems. With the help of proposed algorithm, weights of Functional link artificial neural network were simplified. This algorithm is compared with FLANN, PSO-FLANN and performed better than other two approaches. Shilaskar and Ghatol [5] gave a model which contained the feature selection and the classification techniques. Three data sets from UCI namely Arrhythmia, SPECTF and Heart Disease were used. In this study, SVM classifier was combined with forward feature inclusion, forward feature selection and back-elimination feature selection. The results illustrated that there were reduction in the numbers of inputs and also improvement in the accuracy by using feature selection. For SPECTF dataset, accuracy of SVM increased by $3 \%$ and number of features reduced from 44 to 19 . For heart disease data set, the accuracy of SVM increased by $4 \%$ and number of features reduced from 10 to 4 .

Omar S. Soliman and Eman Abo ElHamd [6] proposed an algorithm based on chaotic levy flights bat algorithm for diagnosing diabetes mellitus. In swarm intelligence, different types of metaheuristics or natured inspired algorithms are present. In this paper, due to the limitation of the bat algorithm authors used the combination of bat algorithm and the chaotic levy flight algorithm. Bat algorithm is based on the echolocation behavior of bats. Due to the searching behavior of bats, there is problem of trapping in local optima and the chaotic variables have set of properties for enhancing the searching behavior that helped in preventing the problem of trapping of Bat algorithm into local optima. The efficiency of getting new solutions was increased through randomization Chances of finding global optimum solutions were also increased. This algorithm was applied algorithm on Pima Indians Diabetes data set and compared with the traditional bat inspired algorithm.

Sarab Al Muhaide and Mohamed El Bachir Menai [7] proposed a hybrid metaheuristic which consists of two steps. The first step was ant colony optimization and the second step was genetic algorithm. In ACO step, by using the different subsets of training data decision lists were made-up. Decision lists were constructed by AntMiner+ algorithm. The population of genetic algorithm was initialized to these decision lists. The genetic algorithm worked for the optimization of these decision lists in term of classification accuracy and size. This algorithm gave the good results on the real world medical data sets.

Mrutyunjaya Panda and Ajith Abraham [8] proposed a novel model for finding the best feature subset with the help of fuzzy rough set based attribute reduction and the nature inspired algorithm ACO and PSO. This paper used the combination of fuzzy rough set with $\mathrm{KNN}$ for classification and applied on the real world datasets. Different tests like Ztest, T-test, sign test and Wilcoxon's signed rank test were used to compare the performance. Among that Wilcoxon's test was better than all other tests.

M. Taha and Y.C.Tang [9] proposed a new optimization algorithm called BAAR. Large data set contains the redundant and irrelevant data and attribute reduction is the process of finding an optimal set of the attribute from the large data set. In this study, authors combined the rough set based attribute reduction with the bat algorithm. The proposed model was compared with the other attribute reduction algorithm and performed better than other algorithms. H. M. Harb and A. S. Desuky [10] used feature Selection based on the Particle Swarm Optimization for the classification of medical data sets. To get the hidden information from the data, different steps are used in data mining. When data contains irrelevant and redundant data, it disturbs the classification accuracy of the data. In this study, the authors used the wrapper and filter feature selection approaches with the particle swarm optimization. The features extracted were applied to the different classifiers like $\mathrm{KNN}$, naïve Bayesian, radial basis function, that improved the classification accuracy as compared to the other techniques like feature selection based on genetic algorithm.

Major findings from the literature review includes that best subset of features are not selected when large number of records. To get the better results of feature selection, accuracy and time, Rough set is used for attribute reduction because it selects subsets of significant attributes. Metaheuristic algorithms are used with rough sets for attribute reduction to find the best minimal attribute reduction.

In this work, Firefly algorithm is used with Levy Flight algorithm. Firefly algorithm was proposed by Yang in 2007 [11], [13] and inspired by the flashing behavior of fireflies. It contains a population-based iterative procedure with many agents (known as fireflies) simultaneously for solving optimization problem [12]. It follows some rules based on the flashing characteristics of real fireflies. Levy Flight algorithm is named for French mathematician Paul Levy. It is a random walk in which steps are defined in terms of step lengths with a certain probability distribution, and with the directions of paces being isotropic and arbitrary [14]. In this research, the moving strategy of firefly uses the levy flight strategy and then combined with the rough sets for attribute reduction. Hence, the main aim of this paper is to recommend an efficient and accurate diagnosis of diseases with reduced number of attributes. In proposed algorithm, the chaos Firefly-Levy Flight algorithm combined with rough set to reduce the set of attributes. The outstanding subsets of features are used as input for the type- 2 fuzzy logic system. The attribute reduction based on rough set using the chaos Firefly-Levy Flight algorithm is compared to the attribute reductions based on rough set using the Chaos Firefly and binary particle swarm optimization. After that the accuracy, sensitivity and accuracy of proposed approach is compared with the other existing techniques.

\section{PROPOSED CHAOS FIREFLY-LEVY FLIGHT ALGORITHM AND ROUGH SET BASED ATTRIBUTE REDUCTION}

The proposed algorithm is the combination of Rough set based Attribute Reduction, Firefly- Levy Flight algorithm and the interval type-2 fuzzy logic. In this method there are three parts (Fig.1): first is normalization, second is attribute reduction which uses the Quick Reduct algorithm, FireflyLevy Flight algorithm and third part is classification process which uses the Interval type-2 fuzzy logic for classification of the reduced data set.

Normalization: The proposed research work uses the minmax normalization which is the popular method of normalization. The min-max normalization is described as [1]: 


$$
v^{\prime}=\frac{v-v_{\min }}{v_{\max }-v_{\min }} * \text { [new_max-new_min] }+ \text { new_min }
$$

Where new_min, new_max is the range of the new dataset which are set to 0 and 1 respectively.

Attribute reduction using chaos firefly-levy flight and rough set: The proposed work computes an attribute reduction using supervised quick reduct algorithm.

Supervised Quick Reduct Algorithm [15]: The supervised feature selection methods are used to estimate different feature subsets with the help of evaluation function to choose only those attributes which are associated with under consideration decision classes of the data [15]. The quick reduct (QR) algorithm calculates a reduct without exhaustively generating all possible subsets [16-18].

Different meta-heuristic algorithms are combined with rough sets to find attribute reduction. These methods use the fitness functions to estimate classification performance and the solution was the maximum value of the fitness function. The value of excellence or quality of approximation of classification in the solution may not equal to 1 . As a result, the solution may not an attribute reduction and also those fitness functions may not consider minimal attribute reduction. To resolve this difficulty, this research proposes chaos firefly-levy flight algorithm and rough sets based attribute reduction. Three important considerations used by the chaos firefly-levy flight algorithm are: 1) Encoding scheme 2) Fitness function 3) Binary code of algorithm for attribute reduction.

In encoding scheme, binary strings are used in which each bit demonstrates an attribute. If the value is 1 then that attribute is selected otherwise not selected.

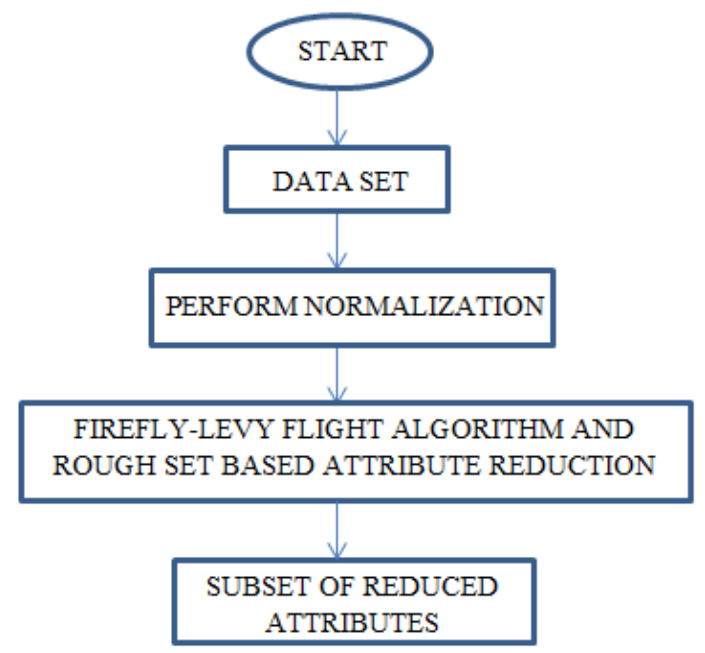

(a) Procedure for Attribute Reduction Step

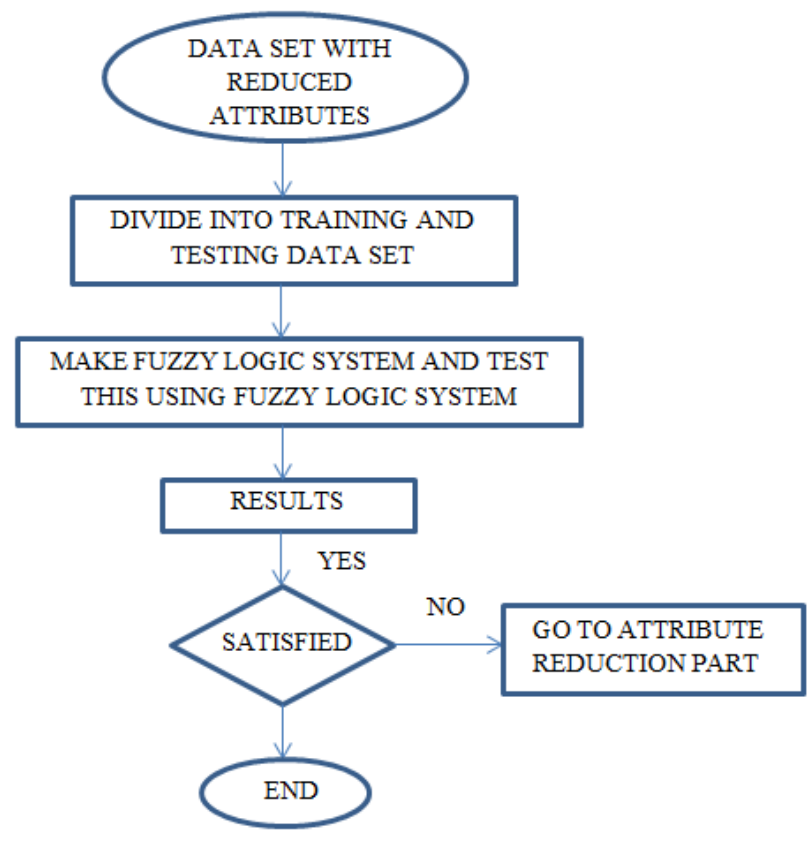

(b) Classification Step of Proposed System

Fig.1 Flow Chart of Proposed Approach for Medical Diagnosis

The fitness function used in this research is as [1]:

$$
\text { Fitness }(X)=\frac{m-|X|}{m}+\frac{n|R| \gamma_{X}(D)}{m \Gamma}
$$

Where $\mathrm{m}=|\mathrm{C}|, \mathrm{n}=|\mathrm{U}|, \gamma_{X}(D)$ is the quality of classification. $\mathrm{R}$ is defined as reduct of condition attributes, $\Gamma=$ $\left|Y_{1}\right|+\left|Y_{2}\right|$ if decision table is consistent otherwise $\Gamma=\left|Y_{1}\right|$.

Binary coding: The 0 and 1 encoding scheme is used in the attribute reduction. It shows that this is a discrete problem. On the other hand, the firefly-levy flight algorithm is used for the continuous problems. To handle this, a tanh function is used which is defines as [1]:

$$
f\left(X_{i}\right)=\frac{\exp \left(2 X_{i}{ }^{k}\right)-1}{\exp \left(2 X_{i}{ }^{k}\right)+1}, \quad \mathrm{i}=1, \ldots, \mathrm{n} ; \mathrm{k}=1, \ldots \ldots, \mathrm{m}
$$

Where $n$ is the number of fireflies in population, $m$ is the number of dimensions.

The working of chaos firefly-levy flight algorithm and rough set based attribute reduction is described below (Algorithm 1). After completing the rough set based attribute reduction using chaos firefly-levy flight algorithm, the minimal subset of attribute is obtained.

These reduced numbers of attributes are divided into the training set and testing set for classification. The training dataset is used to make an model for interval type- 2 fuzzy logic system and this model is test using the testing dataset. 
Algorithm 1: Chaos Firefly-Levy Flight and Rough Set based Attribute Reduction Algorithm

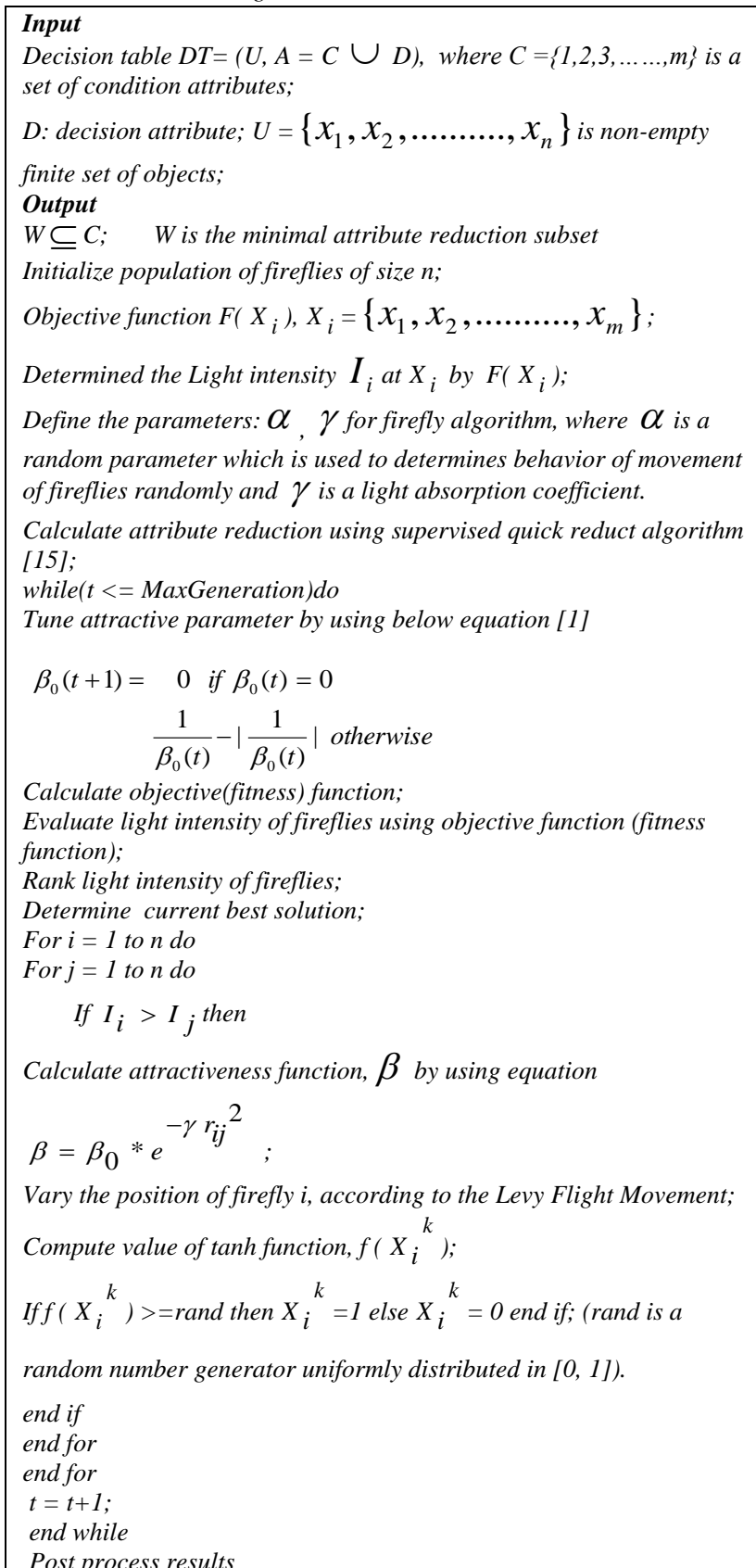

\section{RESULTS AND DISCUSSION}

In this paper, different medical datasets are used for experimental work. The proposed algorithm is implemented in MATLAB and compared with the chaos firefly algorithm, binary particle swarm optimization and rough set based attribute reduction, supervised particle swarm optimization with relative reduct and supervised particle swarm optimization with quick reduct algorithm. For experimental results, five datasets with different types of attributes are chosen from UCI machine repository. A dataset is a collection of data, usually presented in tabular form. Each column is used to represent a particular attribute. Each row represents a given member of the dataset. The description about each dataset is explained below:

Heart disease dataset (catalog): includes the 270 people's records. Each record consists of 13 attributes and one class attribute. The dataset has two classifications, first classifications had 150 records and second one had 120 records.

SPECTF dataset: consists of the 267 images record with 44 attributes and one class attribute in which 55 instances belongs to class 0 and 221 instances to class 1 . Record suggests 80 samples to be used for training (class 0 and class 1 both have 40 samples) and testing data used 187 samples for testing (class 0 have 172 samples and class 1 have 25 samples).

Wisconsin breast cancer diagnosis dataset: This dataset contains 569 samples. The total attributes are 31 and one class attribute. The sample contains 357 benign, 212 malignant. A digitized image of a fine needle aspirate (FNA) of a breast mass computed these features. It explains features of the cell nuclei present in the image.

Prognostic data set: It has total 198 instances and each data has 33 attributes and one class attribute. This data set is computed from a digitized image of a fine needle aspirate (FNA) of a breast mass.

Erythemato-squamous diseases: This data set contains 366 samples and each sample has 33 attributes and one class attribute. The differential diagnosis of erythemato-squamous disease is a real problem in der-matology.

Table 1 gives the description for each of the dataset used for experimental work. This includes Number of instances, number of attributes and number of classes.

Table 1: Description of datasets used for experimental work

\begin{tabular}{|l|l|c|c|c|}
\hline S N & \multicolumn{1}{|c|}{$\begin{array}{c}\text { Name of } \\
\text { Dataset }\end{array}$} & $\begin{array}{c}\text { No. of } \\
\text { Instances }\end{array}$ & $\begin{array}{c}\text { No. of } \\
\text { Attributes }\end{array}$ & $\begin{array}{c}\text { No. of } \\
\text { Classes }\end{array}$ \\
\hline 1. & Heart & 270 & 14 & 2 \\
\hline 2. & SPECTF & 267 & 45 & 2 \\
\hline 3. & Breast Cancer & 569 & 32 & 2 \\
\hline 4. & Prognostic & 198 & 34 & 2 \\
\hline 5. & $\begin{array}{l}\text { Erythemato- } \\
\text { squamous }\end{array}$ & 366 & 34 & 6 \\
\hline
\end{tabular}

Table 2 gives the results of attribute reduction for different types of datasets. According to table 2, the proposed algorithm achieved the minimal number of attribute reductions than the binary particle swarm optimization and chaos firefly algorithm for spectf dataset. Many researchers has been used different algorithms for reducing the feature set.

Table 2: Results After using Attribute Reduction method

\begin{tabular}{|c|c|c|c|c|c|}
\hline $\begin{array}{l}\mathrm{S} \\
\mathrm{N}\end{array}$ & $\begin{array}{l}\text { Name of } \\
\text { Dataset }\end{array}$ & $\begin{array}{l}\text { Number of } \\
\text { Attributes } \\
\text { without } \\
\text { Reduction }\end{array}$ & $\begin{array}{l}\text { Reduced no } \\
\text { of attributes } \\
\text { by } \\
\text { BPSORS- } \\
\text { AR } \\
\text { method }\end{array}$ & $\begin{array}{l}\text { Reduced } \\
\text { no of } \\
\text { attributes } \\
\text { by } \\
\text { CFARS- } \\
\text { AR } \\
\text { method }\end{array}$ & $\begin{array}{l}\text { Reduce } \\
\mathrm{d} \text { no of } \\
\text { attribute } \\
\mathrm{s} \text { by } \\
\text { CFLevy } \\
\text {-AR } \\
\text { method }\end{array}$ \\
\hline 1. & Heart & 14 & 4 & 4 & 4 \\
\hline 2. & $\begin{array}{l}\text { SPECT } \\
\mathrm{F}\end{array}$ & 45 & 4 & 3.64 & 3 \\
\hline
\end{tabular}

Table 3 includes the comparison of proposed algorithm with supervised particle swarm optimization with relative reduct and supervised particle swarm optimization with quick reduct algorithm. It is demonstrated that the CFLevy-AR method has very less number of reduced features than the supervised 
particle swarm optimization with relative reduct and supervised particle swarm optimization with quick reduct method

\begin{tabular}{|c|l|l|l|l|l|}
\hline \multicolumn{6}{|c|}{ Table 3: Comparison with some other existing Attribute } \\
Reduction Method
\end{tabular}

Fig. 2(a) and Fig. 2(b) give the visualization of different types of datasets with reduced number of features through the various reduction algorithms. Fig. 2(a) demonstrates that the proposed algorithm has less number of features than the binary particle swarm optimization and chaos firefly algorithm for SPECTF dataset. Fig. 2(b) represents, the smallest number of reduced features is selected by the proposed algorithm as compared to unreduced data, supervised particle swarm optimization with relative reduct and supervised particle swarm optimization with quick reduct for different datasets.

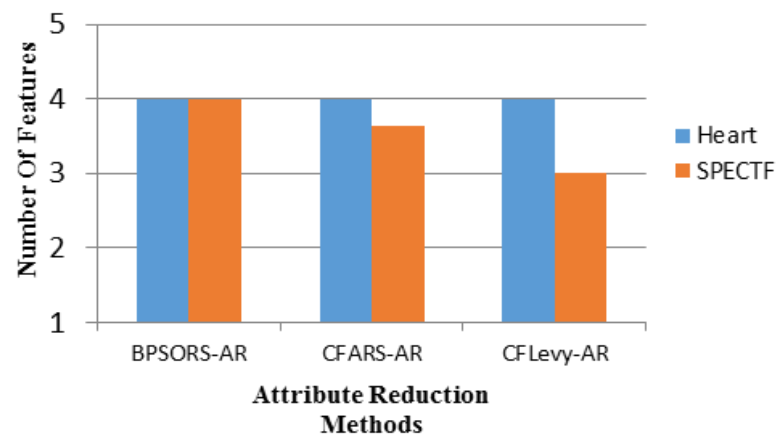

(a) Attribute Reduction for Heart and SPECTF Datasets by Different Methods

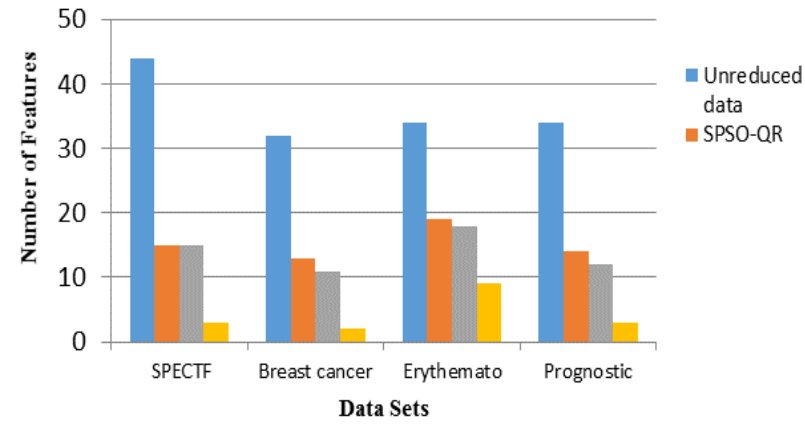

(b)Attribute Reduction for Breast Cancer, Erythemato, Prognostic and SPECTF Datasets by Different Methods

Fig.2 (a)-(b) Comparison of Attribute reduction methods for different datasets

To provide comparison among different classifier, three evaluation measures are used. These are accuracy, sensitivity and specificity. The experimental results of the different classifier with attribute reduction methods are shown in Table 4.

$$
\begin{aligned}
& \text { Accuracy }=\frac{T P+T N}{T P+T N+F P+F N} * 100 \% \\
& \text { Sensitivity }=\frac{T P}{T P+F N} * 100 \% \\
& \text { Specificity }=\frac{T N}{T N+F P} * 100 \%
\end{aligned}
$$

\begin{tabular}{|c|c|c|c|c|c|c|c|c|c|c|c|c|c|}
\hline \multicolumn{14}{|c|}{ Table 4: Performance Comparison } \\
\hline \multirow[t]{2}{*}{ S N } & \multirow[t]{2}{*}{$\begin{array}{c}\text { Name of } \\
\text { Dataset }\end{array}$} & \multicolumn{3}{|c|}{$\begin{array}{l}\text { Without Attribute } \\
\text { Reduction }\end{array}$} & \multicolumn{3}{|c|}{ Using BPSORS-AR } & \multicolumn{3}{|c|}{ Using CFARS-AR } & \multicolumn{3}{|c|}{ Using CFLevy-AR } \\
\hline & & $\%$ Acc & $\%$ Sen & $\%$ Spe & $\%$ Acc & $\%$ Sen & $\%$ Spe & $\%$ Acc & $\%$ Sen & $\%$ Spe & $\%$ Acc & $\%$ Sen & $\%$ Spe \\
\hline 1. & Heart & 86 & 87.1 & 90 & 87.0 & 93.3 & 79.2 & 88.3 & 84.9 & 93.3 & 93 & 100 & 99 \\
\hline 2. & SPECTF & 79.1 & 85.5 & 63.4 & 81.8 & 84.3 & 53.3 & 87.2 & 94.2 & 68.9 & 89.3 & 90.1 & 72.8 \\
\hline
\end{tabular}

From Table 4, it can be seen that the proposed rough set based attribute reduction using levy flight algorithm is better than the without attribute reduction method, binary particle swarm optimization and chaos firefly algorithm in terms of accuracy, sensitivity and specificity for both data sets. 


\begin{tabular}{|c|c|c|c|c|c|c|c|c|c|c|c|c|c|}
\hline \multicolumn{14}{|c|}{ Table 5: Performance Comparison with Some Other Existing Methods } \\
\hline \multirow[t]{2}{*}{$\begin{array}{l}\mathrm{S} \\
\mathrm{N}\end{array}$} & \multirow[t]{2}{*}{$\begin{array}{l}\text { Name of } \\
\text { Dataset }\end{array}$} & \multicolumn{3}{|c|}{$\begin{array}{c}\text { Without Attribute } \\
\text { Reduction }\end{array}$} & \multicolumn{3}{|c|}{ Using SPSO-RR } & \multicolumn{3}{|c|}{ Using SPSO-QR } & \multicolumn{3}{|c|}{ Using CFLevy-AR } \\
\hline & & $\%$ Acc & $\%$ Sen & $\%$ Spe & $\% \mathrm{Acc}$ & $\%$ Sen & $\%$ Spe & $\%$ Acc & $\%$ Sen & $\%$ Spe & $\%$ Acc & \% Sen & $\%$ Spe \\
\hline 1. & $\begin{array}{l}\text { Breast } \\
\text { Cancer }\end{array}$ & 92.28 & 93 & 93 & 93.86 & 93.90 & 94.30 & 94.74 & 94.70 & 94.70 & 95.3 & 95.3 & 95.2 \\
\hline 2. & Prognostic & 92.85 & 95.90 & 86.20 & 92.86 & 92.90 & 86.20 & 92.85 & 95.90 & 86.20 & 72.8 & 93.4 & 0 \\
\hline 3. & Erythemato & 93 & 93 & 94.90 & 97.26 & 97.30 & 94.70 & 97.26 & 97.30 & 94.70 & 98.1 & 94.20 & 96.5 \\
\hline 4 & SPECTF & 79.1 & 85.5 & 63.4 & 88.88 & 88.90 & 88.90 & 83.46 & 83.30 & 83.30 & 89.3 & 90.1 & 72.8 \\
\hline
\end{tabular}

Table 5 gives the comparison of performance of different datasets with other algorithms like unreduced data, supervised particle swarm optimization with relative reduct and supervised particle swarm optimization with quick reduct. It shows that the proposed algorithm has the better performance measures than the other approaches.

Next, there is visualization of comparison of accuracy, sensitivity and specificity for each dataset through the various methods. Fig. 3 demonstrates that an increase in overall accuracy, sensitivity and specificity for different datasets by proposed approach except Prognostic dataset. It reveals the effectiveness of the CFLevy-AR method over the other existing methods.

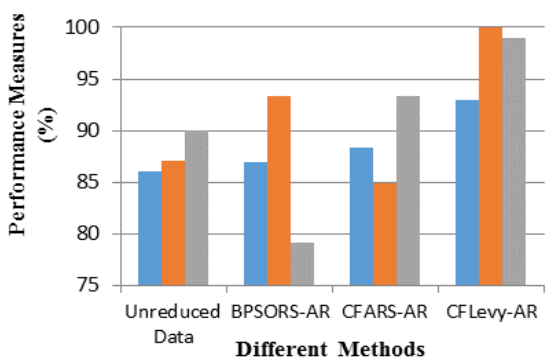

(a) Heart Disease Dataset

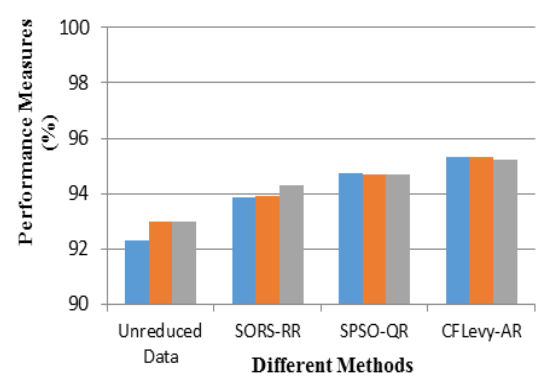

(b) Breast Cancer Dataset

n Accuracy

nenstivity

Specificity

- Accuracy

- Senstivity

- Specificity

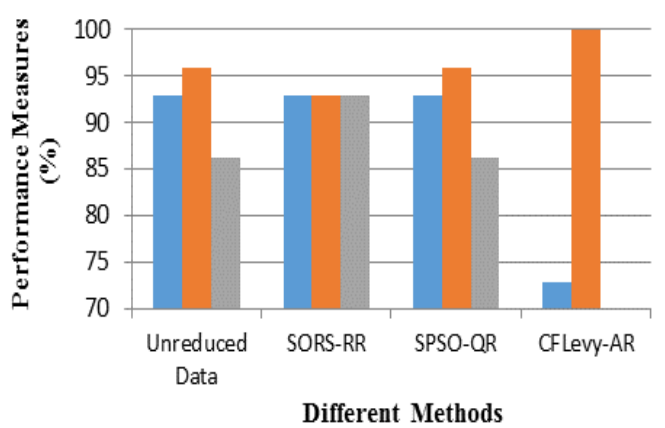

Accuracy

- Senstivity

\secificity

(c)

Prognostic Dataset

है

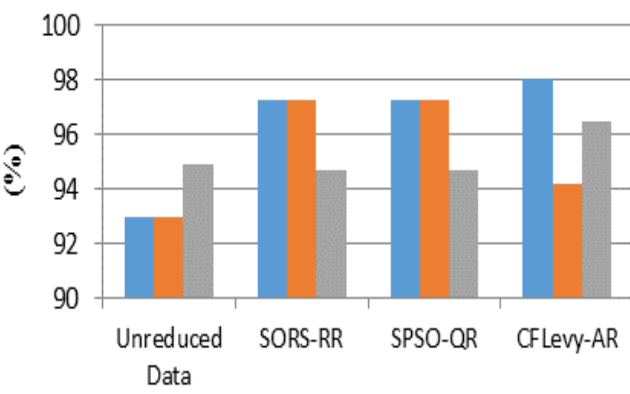

- Accuracy

- Senstivity

-Specificity

Different Methods

(d) Erythemato-squamous Dataset

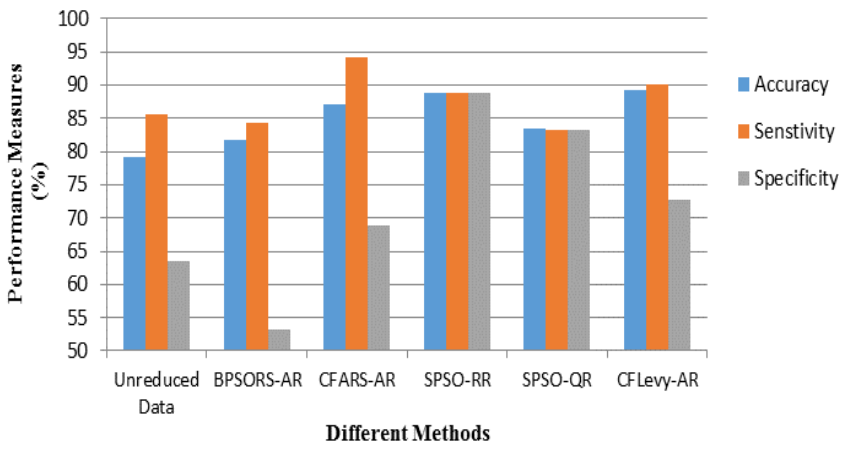

(e) SPECTF Dataset

Fig. 3: (a)-(e) Classification Performance (\%) of Various Medical Data sets 


\section{CONCLUSION}

The proposed algorithm is the combinations of rough set based attribute reduction using firefly-levy flight algorithm and fuzzy logic system for medical diagnosis. It can find the minimum number of attributes efficiently from the high dimensional data sets which is helpful in increasing the performance of the classification system. For handling the uncertainties and noisiness of datasets, fuzzy logic system is successfully implemented. The proposed algorithm on two benchmarked datasets overcomes other methods in terms of accuracy, sensitivity and specificity. Additionally, all the classifier techniques perform better when combined with rough set based attribute reduction method. In particular, the proposed method i.e. firefly-levy flight algorithm gives effective results when combined with fuzzy logic system with fewer features and higher evaluation measures.

In future work, others efficient metaheuristic approaches for attribute reduction will design to manage the large amount of features. Different nature-inspired algorithms will be combined with the different attribute reduction methods and also parallel algorithm will be investigated further for future studies.

\section{REFERENCES}

[1] Long, N. C., Meesad, P. and Unger, H. 2015. A highly accurate firefly based algorithm for heart disease prediction. Expert Systems with Applications, Vol. 42, No. 21, pp. 8221-8231.

[2] Inbarani, H., Azar, A. T. and Jothi, G. 2014. Supervised hybrid feature selection based on PSO and rough sets for medical diagnosis. Computer Methods and Programs in Biomedicine, Vol. 113, No. 1, pp. 175-185.

[3] Bhardwaj, A. and Tiwari, A. 2015. Breast cancer diagnosis using Genetically Optimized Neural Network model. Expert Systems with Applications, Vol. 42, No. 10, pp. 4611-4620.

[4] Mishra, S., Mishra, D. and Shaw, K. 2012. A New Metaheuristic Bat-Inspired Classification Approach for Microarray Data. Procedia Technology, Vol. 4, pp. 802806.

[5] Shilaskar, S. and Ghatol, A. 2013. Feature selection for medical diagnosis: Evaluation for cardiovascular diseases. Expert System with Applications, Vol. 40, No. 10, pp. 4146-4153.

[6] Soliman, O. S. and ElHamd, E Abo. 2015. A Chaotic Levy Flights Bat Algorithm for Diagnosing Diabetes Mellitus. International Journal of Computer Applications, Vol. 111, No. 1, pp. 36-42.
[7] AlMuhaideb, S. and Menai, M. E. B. 2014. A new hybrid metaheuristic for medical data classification. International Journal of Metaheuristics, Vol. 3, No. 1, pp. 59-80.

[8] Panda, M. and Abraham, A. 2014. Hybrid evolutionary algorithms for classification data mining. Neural Computation and Application, Vol. 26, No. 3, pp. 507523.

[9] Taha, A. M. and Tang, Y.C. 2013. Bat Algorithm for Rough Set Attribute Reduction. Journal of Theoretical and Applied Information Technology, Vol. 51, No. 1, pp. 1-8.

[10] Harb, H. M. and Desuky, A. S. 2014. Feature Selection on Classification of Medical Datasets based on Particle Swarm Optimization. International Journal of Computer Applications, Vol. 104, No. 5, pp. 14-17.

[11] Yang, X. S. 2009. Firefly algorithm for multimodal optimization", in proceedings of the stochastic Algorithms. Foundations and Applications (SAGA 109), Vol. 5792 of Lecture notes in Computer Sciences Springer.

[12] Fister, I., Fister, Jr I., Yang, X. S. and Janez, B. 2013. A comprehensive review of firefly algorithms. Swarm and Evolutionary Computation, http://dx.doi.org/10.1016/ j.swevo.2013.06.001.

[13] Yang and Xin-She. 2014. Nature-Inspired Optimization Algorithms. in Nature-Inspired Optimization Algorithms, Oxford: Elsevier, p. iii.

[14] Kamaruzaman, A.F., Zain, A. M., Yusuf, S. M. and Udin A. 2013. Levy Flight Algorithm for Optimization Problems - A Literature Review. Applied Mechanics and Materials, Vol. 421, pp 496-501.

[15] Velayutham, C. and Thangavel, K. 2011. Unsupervised quick reduct algorithm using rough set theory. Journal of Electronic Science and Technology (JEST) (International), Vol. 9, No. 3, pp. 193-201.

[16] Jensen, R. and Shen, Q. 2004. Semantics-preserving dimensionality reduction: rough and fuzzy-rough based approaches. IEEE Transaction. On Knowledge and Data Engineering, Vol. 16, No. 12, pp. 1457-1471.

[17] Jensen, R. and Shen, Q. 2004. Fuzzy-rough attribute reduction with application to web categorization. Fuzzy Sets and Systems, Vol. 141, No. 3, pp. 469-485.

[18] Jensen, R. 2004. Combining rough and fuzzy sets for feature selection. Ph.D. Dissertation, School of Informatics, University of Edinburgh, Edinburgh 